TuB2.7 (Postdeadline)

3:15 pm - 3:30 pm

\title{
Pure luminescence transitions from a small InAs/GaAs quantum dot exhibiting a single electron level
}

\author{
K. F. Karlsson ${ }^{1}$, P. O. Holtz ${ }^{1}$, E. S. Moskalenko ${ }^{1}$, B. Monemar ${ }^{1}$ \\ W. V. Schoenfeld ${ }^{2}$, J. M. Garcia ${ }^{2}$ and P. M. Petroff ${ }^{2}$ \\ 'Department of Physics, Linköping University, S- 58183 Linköping, Sweden \\ ${ }^{2}$ Materials Department, University of California, Santa Barbara, California 93106 \\ Phone: $+46(0) 13282628$ Fax: +46(0)13142337 E-mail: poh@ifm.liu.se
}

Pure photoluminescence spectra originating from a single InAs/GaAs quantum dot, which is small enough to possess only one single-electron level, are demonstrated. A symmetric fine structure of the exciton and the biexciton is observed.

Carriers confined in a quantum dot (QD) are strongly interacting, which makes the energy states very sensitive to the exact number of trapped carriers. In fact, one single extra carrier is enough to significantly change the electrical and optical behavior of the QD. Therefore, in the moderate-excitation-power regime, the QDs is on average populated with more than one exciton and several emission lines appear in the photoluminescence (PL) spectra, reflecting the increased number of possible carrier configurations. The appearance of the biexciton, which shows that the first electron and hole levels are filled, is normally associated with a complex set of emission lines originating from the decay of multiexciton complexes involving also higher electron- and hole levels (see figure 1). Also the appearance of doubly charged excitons or charged biexcitons proves the involvement of higher energy levels.

The simplest QD with the ability to confine excitons possesses one single electron and one single hole level, which can accommodate at maximum two electrons and two holes, respectively. In addition to the single exciton, such a QD can confine a very limited number of complexes in comparison with the multilevel QD: A negatively/positively charged exciton or a biexciton.

A micro-PL spectrum of a single QD with at least three electron and hole levels is shown in Fig.1. The excitation creates electrons and holes in the GaAs barrier with a power that is chosen in such a way that an essential contribution originates from the biexciton (2X) while the single exciton (X) dominates the spectrum. The low-energy side of $2 \mathrm{X}$ has a rich structure of emission lines originating from higher orders of exciton complexes including charged excitons. In addition to the recombination of electrons and holes both in the lowest energy level $(s \rightarrow s)$, a contribution related to higher energy levels is observed $(p \rightarrow p)$. The inset of Fig. 1 exhibits a spectrum of negatively charged excitons, when the QDs is charged with one $\left(\mathrm{X}^{-}\right)$or two $\left(\mathrm{X}^{-}\right)$extra electrons [1-2].

Fig. 2 shows analogous spectra of another QD for which no $p \rightarrow p$ transitions exist. The absence of the higher levels makes the spectrum much more pure and essentially all emission lines below $2 \mathrm{X}$ vanish. When the QDs is charged by extra electrons [1-2], only the $\mathrm{X}^{-}$is exhibited (se the inset of Fig. 2) while the $\mathrm{X}^{-}$is absent. Consequently, only one single electron levels exist in this particular QD. The very extraordinary pureness of the spectra of QDs without excited levels makes them interesting for studies of the exciton fine structure.

The $\mathrm{X}$ and $2 \mathrm{X}$ in Fig. 2 exhibit shoulders on the high- and the low-energy side, respectively. In fact, the shoulders consists of a set of lines (four lines have been observed altogether) which relative intensities change with the sample temperature or excitation energy 
and power. One explanation for this fine structure could be the splitting of the exciton emission into four different components caused by the mixing of the bright and dark excitons in asymmetric QDs [3]. The mixing relies on the electron-hole exchange interaction which is expected to be strong in small QDs. The initial state of the biexciton $(2 \mathrm{X})$ recombination is a singlet state, which consequently is unsplit, while the final state of the biexciton decay is the split single exciton (X). Accordingly, the relative energies of the emission lines associated with the biexction simply correspond to the reversed relative energies of the exciton (see the schematic decay diagram in the left part of Fig. 3). In fact, such a reversed energy relation can be observed when the excitation conditions are adjusted and three peaks of the fine structure are clearly revealed. For clarity, the exciton emission is shown together with the mirrored and translated biexciton emission in Fig. 3. A good agreement in the peak energy positions between the $\mathrm{X}$ and the mirrored $2 \mathrm{X}$ is obtained. No splitting is observed for the negatively charged exciton and no exchange splitting is expected due to the singlet nature of the electron pair [3].

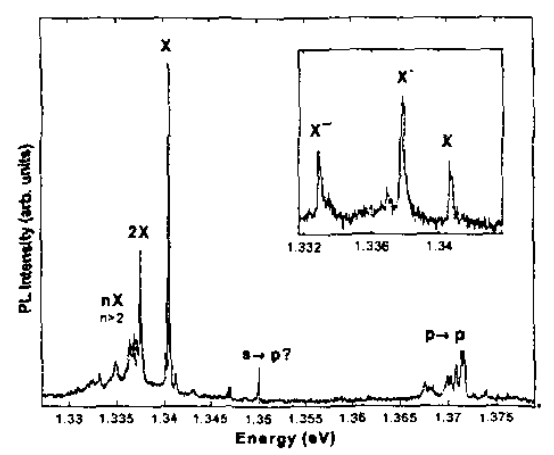

Figure 1. Micro-PL spectra of a QD exhibiting multiple energy levels for both electrons and holes. The inset shows a spectrum of the same QD charged with zero, one or two extra electrons.

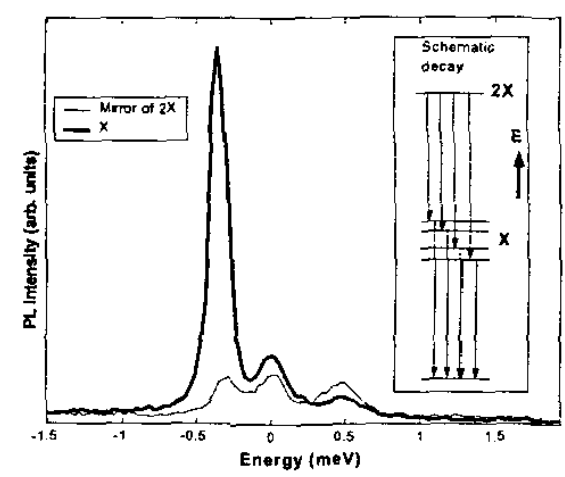

Figure 3. The exciton $(X)$ emission from a $Q D$ and the mirrored and translated biexction emission $(2 X)$. The inset shows schematically the decays from $2 X$ to the splitted $X$ and subsequently from $X$ to an empty $Q D$.

\section{References}

E. S. Moskalenko et al. Phys. Rev. B 64, 085302 (2001)

E. S. Moskalenko et al. Phys. Rev. B 66, 195332 (2002)

M. Bayer et al. Phys. Rev. B 65, 195315 (2002)

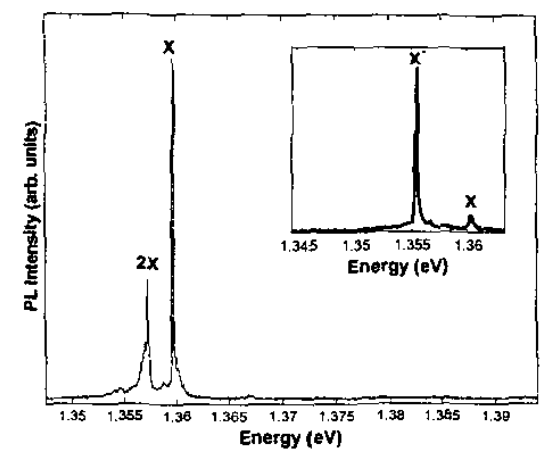

Figure 2. Micro-PL spectra of a QD exhibiting a single electron energy level. The inset shows spectra of the same $\mathrm{QD}$ charged with mainly one extra electron. 